\title{
Trypanosoma cruzi and Toxoplasma gondii Induce a Differential MicroRNA Profile in Human Placental Explants
}

\section{OPEN ACCESS}

Edited by:

Adrian John Frederick Luty, Institut de Recherche Pour le Développement (IRD), France

Reviewed by: Dolores Correa, National Institute of Pediatrics (Mexico), Mexico

Marisa Mariel Fernandez, Institute of Studies on Humoral Immunity (IDEHU), Argentina

*Correspondence: Ulrike Kemmerling ukemmerling@uchile.cl

Specialty section:

This article was submitted to Microbial Immunology, a section of the journal Frontiers in Immunology

Received: 15 August 2020 Accepted: 12 October 2020 Published: 06 November 2020

Citation:

Medina L, Castillo C, Liempi A, Guerrero-Muñoz J, Rojas-Pirela M, Maya JD, Prieto $H$ and Kemmerling $U$ (2020) Trypanosoma cruzi and Toxoplasma gondii Induce a Differential MicroRNA Profile in Human Placental Explants.

Front. Immunol. 11:595250. doi: 10.3389/fimmu.2020.595250

\begin{abstract}
Lisvaneth Medina $^{1}$, Christian Castillo ${ }^{1}$, Ana Liempi ${ }^{1}$, Jesús Guerrero-Muñoz ${ }^{1}$, Maura Rojas-Pirela ${ }^{2}$, Juan Diego Maya ${ }^{3}$, Humberto Prieto ${ }^{4}$ and Ulrike Kemmerling ${ }^{1 *}$

1 Programa de Anatomía y Biología del Desarrollo, Facultad de Medicina, Instituto de Ciencias Biomédicas, Universidad de Chile, Santiago, Chile, 2 Instituto de Biología, Pontificia Universidad Católica de Valparaíso, Valparaíso, Chile, ${ }^{3}$ Programa de Farmacología Molecular y Clínica, Facultad de Medicina, Instituto de Ciencias Biomédicas, Universidad de Chile, Santiago, Chile, ${ }^{4}$ Instituto de Investigaciones Agropecuarias, Ministerio de Agricultura, Santiago, Chile
\end{abstract}

Trypanosoma cruzi and Toxoplasma gondii are two parasites than can be transmitted from mother to child through the placenta. However, congenital transmission rates are low for $T$. cruzi and high for $T$. gondii. Infection success or failure depends on complex parasite-host interactions in which parasites can alter host gene expression by modulating non-coding RNAs such as miRNAs. As of yet, there are no reports on altered miRNA expression in placental tissue in response to either parasite. Therefore, we infected human placental explants ex vivo by cultivation with either T. cruzi or T. gondii for $2 \mathrm{~h}$. We then analyzed the miRNA expression profiles of both types of infected tissue by miRNA sequencing and quantitative PCR, sequence-based miRNA target prediction, pathway functional enrichment, and upstream regulator analysis of differentially expressed genes targeted by differentially expressed miRNAs. Both parasites induced specific miRNA profiles. GO analysis revealed that the in silico predicted targets of the differentially expressed miRNAs regulated different cellular processes involved in development and immunity, and most of the identified KEGG pathways were related to chronic diseases and infection. Considering that the differentially expressed miRNAs identified here modulated crucial host cellular targets that participate in determining the success of infection, these miRNAs might explain the differing congenital transmission rates between the two parasites. Molecules of the different pathways that are regulated by miRNAs and modulated during infection, as well as the miRNAs themselves, may be potential targets for the therapeutic control of either congenital Chagas disease or toxoplasmosis.

Keywords: Trypanosoma cruzi, Toxoplasma gondii, human placental explants, miRNA profile, host gene expression 


\section{INTRODUCTION}

More than one billion people worldwide are burdened by parasitic diseases (1). Of these, Chagas disease (American trypanosomiasis) and toxoplasmosis are caused by Trypanosoma cruzi (T. cruzi) and Toxoplasma gondii ( $T$. gondii), respectively (2-4). Chagas disease is a devastating but neglected health problem in Latin America. Due to the extensive global migration of asymptomatic individuals, this infection has become an emerging disease in non-endemic countries. Congenital transmission is partially responsible for the progressive globalization of Chagas disease $(5,6)$. T. gondii is one of the most successful parasites on earth and is estimated to infect over one billion people worldwide (7). Importantly, both parasites can be congenitally transmitted and cause perinatal morbidity and mortality (2-4) but present different transmission rates. T. cruzi has a low transmission rate (1-12\%) $(6,8)$ while $T$. gondii has a high transmission rate (22-72\%) (3). Moreover, both parasites elicit a different local placental immune response that might be related to infection susceptibility $(9,10)$. Thus, $T$. cruzi and $T$. gondi infection is related to the expression and activation of different Toll-like receptors, which in turn mediate the secretion of different cytokines and chemokines in defense against both parasites in the placenta $(9,11)$.

However, the probability of congenital transmission depends on a variety of complex interactions between the pathogen and the host $(4,12)$. In particular, parasite factors, placental factors, and maternal and developing fetal immune systems determine infection occurrence $(4,13)$. In this context, both parasites display sophisticated strategies to avoid host defenses and virulence factors that increase the chance of establishing infection and long-term persistence. One of these strategies is the ability to modulate host cell gene expression (14-16) through small non-coding RNAs such as microRNAs (miRNAs) that repress mRNAs in a sequence-specific manner by either an mRNA degradation process or through mRNA translation inhibition (17-19). MiRNAs play a key role in fine tuning gene expression in multiple physiological and pathological conditions including T. cruzi (20) or T. gondii (21) infection. Interestingly, the largest miRNA cluster in humans is encoded in chromosome 19 (C19MC; 19q13.41) and is almost exclusively expressed in the placenta (22). Both C19MC-derived and non-C19MC-derived miRNAs have been associated with placental development pathologies such as pre-eclampsia and infection $(23,24)$. However, there is no report in the literature regarding altered miRNA expression in placental tissue in response to either parasite.

Here, we infected human placental explants (HPE) ex vivo by $2 \mathrm{~h}$ of incubation with either T. cruzi or T. gondii, then analyzed both miRNA expression profiles by miRNA sequencing and quantitative PCR of selected miRNAs. In addition, we used sequence-based miRNA target prediction and performed pathway functional enrichment and upstream regulator analysis of differentially expressed genes targeted by differentially expressed miRNAs (DEMs).

\section{MATERIALS AND METHODS}

\section{Parasite Culture and Harvesting}

For T. cruzi, Y strain (T. cruzi II) trypomastigotes were obtained from previously infected Vero cells (ATCC ${ }^{\circledR}$ CCL-81) grown in RPMI medium supplemented with $5 \%$ fetal bovine serum and $1 \%$ antibiotics (penicillin-streptomycin) at $37^{\circ} \mathrm{C}$ in a humid atmosphere with $5 \% \mathrm{CO}_{2}$. Parasites invaded the cells and replicated intracellularly as amastigotes. After 48-72 h, amastigotes transformed back to trypomastigotes and lysed the host cells. The infective trypomastigotes were separated from cellular debris by low speed centrifugation $(500 \times g)$ for $10 \mathrm{~min}$. Parasites were isolated from the supernatant by centrifugation at $3500 \times g$ during $15 \mathrm{~min}$, suspended in RPMI media (without fetal bovine serum, 1\% (penicillin-streptomycin) (RPMI $1640^{\circledR}$, Biological Industries Ltd.), and quantified in a Neubauer chamber (9).

For T. gondii, semi-confluent HFF cells were infected with $\mathrm{RH}$ tachyzoites at a multiplicity of infection of 3 to 5 parasites per cell. After $40 \mathrm{~h}$, the infected cells were washed, then monolayers were scraped from the flasks and passed through 20-, 23-, and 25 -gauge needles. Tachyzoites were purified from host cell debris with a $3.0 \mu \mathrm{m}$ Isopore filter (Merck Millipore ${ }^{\circledR}$ ) (25).

The laboratory has been certificated as a Biosafety level 2 laboratory by the Biosafety Committee ("Unidad de Prevención de Riesgo") of the "Facultad de Medicina, Universidad de Chile" (approval \# 0403/2019).

\section{HPE Infection}

Human term placentas were obtained from 3 women with uncomplicated pregnancies with vaginal or caesarean delivery. Informed consent for experimental use of the placenta was given by each patient as stipulated by the Code of Ethics of the "Servicio de Salud Metropolitana Norte" (approval number 0010/2019). Exclusion criteria consisted of the following: major fetal abnormalities, placental tumor, intrauterine infection, obstetric pathology, positive serology for Chagas disease, and any other maternal disease. Donor patients were negative for anti-T. gondii IgG/IgM antibodies. The organs were collected in cold, sterile, saline-buffered solution (PBS) and processed no more than $30 \mathrm{~min}$ after delivery. The dissected explants (approximately $50 \mathrm{mg}$ of tissue) were washed with sterile PBS to remove the blood and co-cultivated with T. cruzi trypomastigotes or T. gondii tachyzoites $\left(10^{5}\right.$ parasites $\left./ \mathrm{ml}\right)$ in serum free RPMI media. After $2 \mathrm{~h}$ of co-cultivation, explants were collected in RNAlater ${ }^{\mathrm{TM}}$ solution (ThermoFisher Scientific ${ }^{\circledR}$ ), then stored at $4^{\circ} \mathrm{C}$ for $24 \mathrm{~h}$ and at $-80^{\circ} \mathrm{C}$ for posterior RNA isolation (9). Three independent experiments were carried out in triplicates; HPEs from each placenta were infected with either T. cruzi or T. gondii parasites. The parasite load in the HPEs was confirmed by real-time PCR as described previously by us $(9,10,26)$.

\section{RNA Extraction}

Total RNA was extracted from HPE by mechanical disruption in $1.3 \mathrm{ml}$ of RNA-solv ${ }^{\circledR}$ reagent (Omega Bio-tek) and isolated using 
an E.Z.N.A ${ }^{\circledR}$ total RNA kit I (Omega Bio-tek) according to manufacturer instructions. RNA was stored at $-80^{\circ} \mathrm{C}$ until analysis. The concentration and quality of RNA was determined with a Qubit ${ }^{\circledR}$ RNA HS Assay kit and an IQ Assay kit (Invitrogen), respectively. Only RNA samples with an IQ $\geq 8$ were further analyzed for quality with an Agilent 2100 Bioanalyzer System (Agilent Technologies, USA) using an RNA Nano 6000 Assay Kit. RNA samples with RNA integrity numbers $>5.0$ were used for miRNA profiling analysis (9).

\section{Library Construction and Sequencing}

Small RNA-Seq libraries were constructed with an Illumina TruSeq Small RNA library preparation kit according to manufacturer protocols. To assess the quality of the libraries, a DNA High Sensitivity Chip was used in an Agilent 2100 Bioanalyzer (Agilent Technologies, USA). The libraries were sequenced on an Illumina NextSeq 500 platform. For each condition, three independent biological replicates were sequenced and paired-end reads were generated.

\section{Data Analysis}

Raw read quality was evaluated using the FastQC tool (https://www. bioinformatics.babraham.ac.uk/projects/fastqc/). Raw reads were analyzed with Trim Galore Cutadapt software (27) and lowquality reads were removed (phred value $<30$ ) in order to obtain clean reads. Clean reads with a length range of 18-35 nucleotides were chosen to perform all subsequent analyses. The sofware STAR (28) was used to align all reads to the reference human genome sequence $(\mathrm{Hg} 38)$. The read counts per coding sequence were determined using HTSeq-count (29). To evaluate replicates, we used Principal Component Analysis, Pearson correlation and standardized median correlation analyses and box plots. The program EdgeR was used for differential expression analysis (30). Differentially expressed genes were defined as genes with p-value $<0.05$. Target gene prediction performed by using miRDB, psRNA target, and TargetScan sofwares.

\section{Enrichment Analyses}

miRNA set enrichment analysis was performed using the TAM 2.0 tool (http://www.lirmed.com/tam2/). KEGG pathways and functional annotation of the predicted target genes (https://www. genome.jp/kegg/kegg2.html) were also analyzed to determine the biological processes, molecular functions, cellular components, and associations with disease.

\section{RT-qPCR}

RNA enriched in small RNAs was extracted from HPEs (approximately $50 \mathrm{mg}$ of tissue) by mechanical disruption in $1 \mathrm{ml} \mathrm{RNAzol}{ }^{\circledR}$ RT (Sigma-Aldrich) according to manufacturer instructions and stored at $-80^{\circ} \mathrm{C}$ until analysis. The concentration of the isolated miRNAs was determined using a Qubit $^{\circledR}$ Quant-iT ${ }^{\mathrm{TM}}$ microRNA Assay Kit (Molecular Probes). cDNA of mature miRNAs was synthesized with a MystiCq ${ }^{\mathrm{TM}}$ microRNA cDNA Synthesis Mix Kit (Sigma-Aldrich Merck) per manufacturer guidelines. The $25 \mu \mathrm{l}$ RT-qPCR reaction contained $12.5 \mu \mathrm{l} 2 \times$ MystiCq microRNA SYBR Green qPCR Ready Mix, $0.5 \mu \mathrm{l}$ of $10 \mu \mathrm{M}$ MystiCq Universal PCR Primer, $0.5 \mu \mathrm{l}$ of $10 \mu \mathrm{M}$ of each specific MystiCq microRNA qPCR Assay Primer (Supplementary Table 1), $10.5 \mu \mathrm{l}$ nuclease-free water, and $1 \mu \mathrm{l}$ cDNA. All RT-qPCR reactions were performed in triplicates. RT-qPCR was performed under the following cycling conditions: initial denaturation at $95^{\circ} \mathrm{C}$ for $2 \mathrm{~min}$, followed by 40 cycles of $95^{\circ} \mathrm{C}$ for $5 \mathrm{~s}$ and $60^{\circ} \mathrm{C}$ for $30 \mathrm{~s}$. Gene expressions were calculated using the $2^{-\Delta \Delta \mathrm{CT}}$ relative expression method and normalized to snRNA U6 (RNU6-1) expression levels (31).

\section{RESULTS}

\section{T. cruzi and T. gondii Change the miRNA Expression Profile in HPE}

The effects of $T$. cruzi and T. gondii on placental tissue were assayed in HPE after a $2 \mathrm{~h}$ challenge with $10^{5}$ parasites $/ \mathrm{ml}$. Total miRNA extracted from infected and non-infected control HPE was analyzed by miRNA Seq. Key characteristics of the obtained sequencing data are summarized in Table 1. A total of 680 and 686 DEMs were identified in T. cruzi and T. gondii infected HPE, respectively. Only 14 DEMs with a minimum 1.5 -fold change in expression and a $95 \%$ probability of being differentially expressed ( $\mathrm{p} \leq 0.05$ ) were identified in T. cruzi challenged samples (Figure 1A). In $T$. gondii challenged samples, the number of DEMs increased to 42 (Figure 1B). Comparison of T. cruzi infected HPE with non-infected control samples showed that five

TABLE 1 | Statistics of the small RNA sequences obtained in this study.

\begin{tabular}{|c|c|c|c|c|c|c|}
\hline & M reads (millions) & M Aligned (millions) & $\%$ aligned & Mature microRNA reads & \# Mature MicroRNAs & \# Mature MicroRNA (single aligned) \\
\hline S1 Control & 12,96 & 11,68 & 90,12 & 3489287 & 922 & 732 \\
\hline S2 Control & 15,17 & 13,87 & 91,43 & 4855933 & 938 & 760 \\
\hline S3 Control & 15,58 & 13,16 & 84,50 & 3030989 & 843 & 714 \\
\hline S4 T. cruzi & 14,35 & 12,60 & 87,77 & 3000672 & 907 & 712 \\
\hline S5 T. cruzi & 14,76 & 12,94 & 87,69 & 3910944 & 907 & 737 \\
\hline S6 T. cruzi & 16,13 & 13,15 & 81,55 & 3571933 & 914 & 720 \\
\hline S7 T. gondii & 13,57 & 11,82 & 87,07 & 3160630 & 916 & 737 \\
\hline S8 T. gondii & 14,40 & 12,79 & 88,77 & 4747358 & 962 & 757 \\
\hline S9 T. gondii & 13,51 & 12,16 & 89,99 & 4148647 & 931 & 763 \\
\hline
\end{tabular}

$M$ reads, total of sequences in analysis after raw data processing (millions); M aligned, total of mapped sequences to the human genome (millions); \% aligned, total of mapped sequences to the human genome (percentage); Mature microRNA reads, mapped reads counted as mature miRNAs; \# Mature microRNAs, number of mature miRNAs; \# Mature MicroRNA (single aligned), number of mature miRNAs associated to unique miRNA precursors in the reference. 

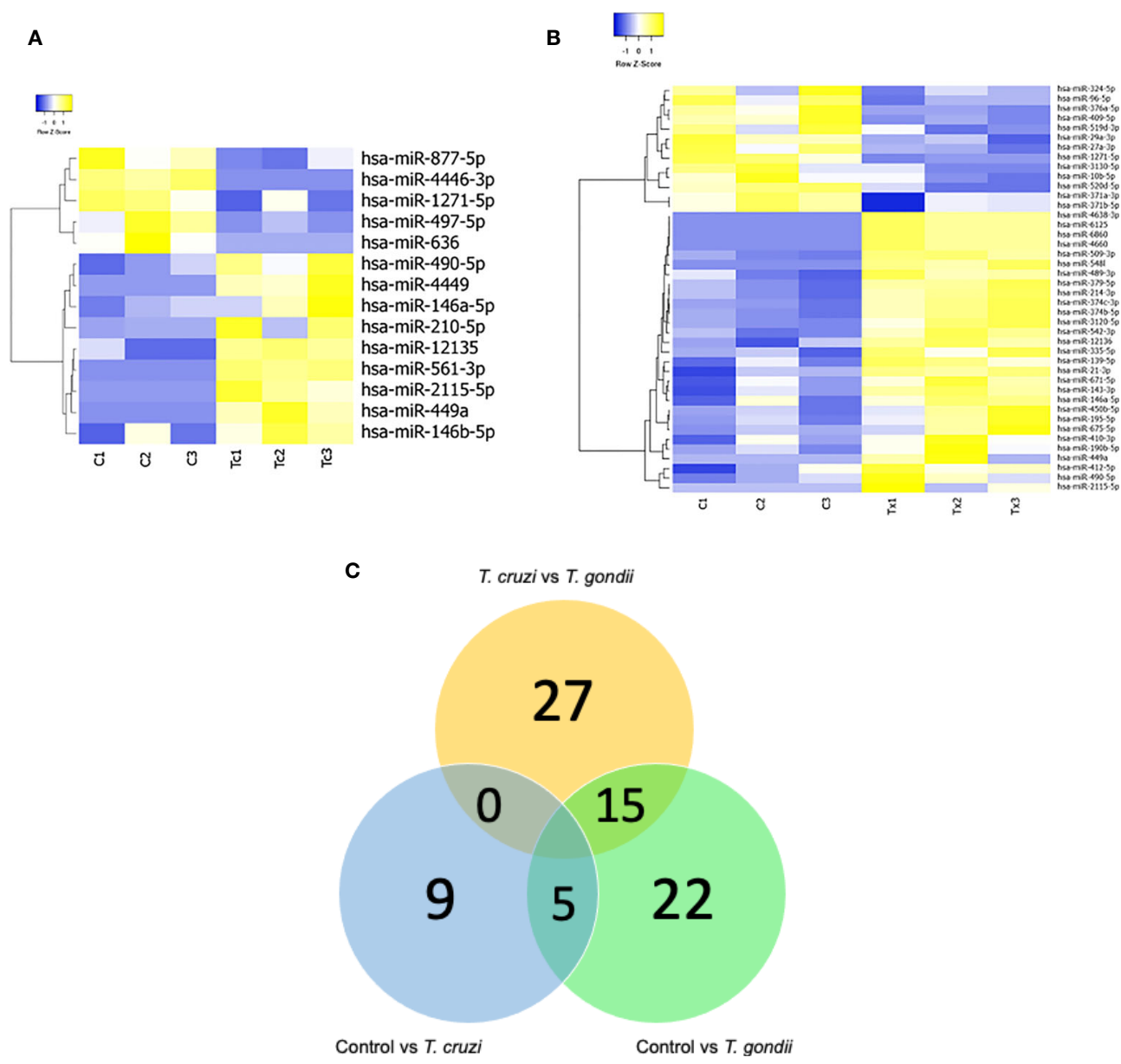

FIGURE 1 | Expression profiling of mature miRNAs in human placental explants (HPEs) following T. cruzi or T. gondii infection. (A) Heat-map of differentially expressed microRNAs (DEMs) in control vs. T. cruzi infected HPE and (B) control vs. T. gondii infected HPE. The filtered miRNA data were subjected to unsupervised hierarchical clustering analysis. The metric was set as the Euclidean distance. Control: C1, C2, C3; T. cruzi: Tc1, Tc2, Tc3; T. gondii: Tx1, Tx2, Tx3. (C) Venn diagram showing the number of commonly expressed and specifically expressed miRNAs between the infected HPE groups. Significant miRNAs for HPE with $T$ cruzi infection vs. those with $T$. gondii infection are shown in the yellow circle. The blue circle represents the miRNAs that discriminate between the control (uninfected HPE) and T. cruzi infected HPE, while the green circle represents the miRNAs that distinguish the control (uninfected HPE) and T. gondii infected HPE.

miRNAs were downregulated and nine were upregulated. In $T$. gondii infected tissues, 13 miRNAs were downregulated and 29 were upregulated. The Venn diagram in Figure 1C shows the miRNAs that were differentially expressed in the presence of both parasites compared to non-infected control samples and in HPE infected with either parasite. The complete list of DEMs in response to ex vivo T. cruzi and T. gondii infection is shown in Table 2.

\section{Functional Annotation and KEGG Pathway Enrichment Analysis of miRNA Target Genes}

To better understand the roles of the miRNAs identified in HPE in response to ex vivo infection with both parasites, the target genes of the miRNAs were identified using miRDB, psRNA target, and TargetScan. GO and KEGG enrichment analyses used to identify the biological functions of the DEMs ( $\mathrm{p}<$ 0.05) during ex vivo T. cruzi and T. gondii infection revealed
679 best scored target genes of the 14 miRNAs from T. cruzi vs. control, 1970 best scored target genes of the 42 miRNAs from $T$. gondii vs. control, and 2011 best scored target genes of the 42 miRNAs from T. cruzi vs. T. gondi. The target genes of the DEMs are shown in Supplementary Table 2. Among the significantly enriched GO terms in T. cruzi vs. control samples, DEMs were significantly enriched in regulation of $\mathrm{NF \kappa B}$ pathways, chondrocyte development, cell death including apoptosis, peritoneal cavity homeostasis, angiogenesis, cell cycle, megakaryocyte differentiation, Toll-like receptor signaling pathway, and immune response including innate immunity (Figure 2A). Among the significantly enriched GO terms in $T$. gondii vs. control samples, DEMs were significantly enriched in cell proliferation, cell migration, osteoblast differentiation, oxidative stress, lipid metabolism, regulation of stem cells including embryonic stem cells, hepatotoxicity, DNA damage response, regulation of $\mathrm{NF \kappa B}$ pathways, smooth muscle 
TABLE 2 | The top differentially expressed miRNAs $(P<0.05)$ in HPE challenged with $10^{5}$ T. cruzi trypomastigotes or T. gondii tachyzoites during 2 h.

\begin{tabular}{|c|c|c|c|c|c|}
\hline & miRNAs & Fold Change & p-value & p-adjustment & Expression \\
\hline \multirow[t]{8}{*}{ Control vs T. cruzi } & hsa-miR-490-5p & 1,47644601 & 0,011939305 & 1 & Up-regulated \\
\hline & hsa-miR-497-5p & $-0,95822045$ & 0,021380502 & 1 & Down-regulated \\
\hline & hsa-miR-146a-5p & 0,9111643 & 0,024398238 & 1 & Up-regulated \\
\hline & hsa-miR-12135 & 2,76826832 & 0,025125553 & 1 & Up-regulated \\
\hline & hsa-miR-210-5p & 1,17471038 & 0,038576256 & 1 & Up-regulated \\
\hline & hsa-miR-146b-5p & 0,70985057 & 0,041583753 & 1 & Up-regulated \\
\hline & hsa-miR-877-5p & $-0,71572824$ & 0,049301458 & 1 & Down-regulated \\
\hline & hsa-miR-1271-5p & $-1,48497011$ & 0,049553349 & 1 & Down-regulated \\
\hline \multirow[t]{6}{*}{ T. cruzi ('treatment exclusive) } & hsa-miR-636 & & 0,003652701 & 1 & Up-regulated \\
\hline & hsa-miR-4449 & & 0,005197851 & 1 & Up-regulated \\
\hline & hsa-miR-449a & & 0,009529598 & 1 & Up-regulated \\
\hline & hsa-miR-2115-5p & & 0,013101594 & 1 & Up-regulated \\
\hline & hsa-miR-561-3p & & 0,023021063 & 1 & Up-regulated \\
\hline & hsa-miR-4446-3p & & 0,041796581 & 1 & Up-regulated \\
\hline \multirow[t]{35}{*}{ Control vs T. gondii } & hsa-miR-12136 & 1,79576197 & 0,00017626 & 0,120736654 & Up-regulated \\
\hline & hsa-miR-335-5p & 1,35817451 & 0,0007489 & 0,227732906 & Up-regulated \\
\hline & hsa-miR-10b-5p & $-1,51399053$ & 0,00099737 & 0,227732906 & Down-regulated \\
\hline & hsa-miR-1271-5p & $-2,41452099$ & 0,00206894 & 0,267855922 & Down-regulated \\
\hline & hsa-miR-409-5p & $-2,51430487$ & 0,00225422 & 0,267855922 & Down-regulated \\
\hline & hsa-miR-27a-3p & $-0,97356696$ & 0,00234618 & 0,267855922 & Down-regulated \\
\hline & hsa-miR-29a-3p & $-0,92877383$ & 0,00344997 & 0,337603687 & Down-regulated \\
\hline & hsa-miR-214-3p & 0,9642214 & 0,00435186 & 0,372627686 & Up-regulated \\
\hline & hsa-miR-379-5p & 1,46136088 & 0,00619518 & 0,453658878 & Up-regulated \\
\hline & hsa-miR-3120-5p & 1,05085403 & 0,00662276 & 0,453658878 & Up-regulated \\
\hline & hsa-miR-376a-5p & $-1,06317073$ & 0,00928715 & 0,578335925 & Down-regulated \\
\hline & hsa-miR-542-3p & 1,36466429 & 0,01456701 & 0,760274481 & Up-regulated \\
\hline & hsa-miR-195-5p & 1,29637989 & 0,01520981 & 0,760274481 & Up-regulated \\
\hline & hsa-miR-3130-5p & $-2,64645685$ & 0,01847345 & 0,760274481 & Down-regulated \\
\hline & hsa-miR-519d-3p & $-0,91993812$ & 0,0188483 & 0,760274481 & Down-regulated \\
\hline & hsa-miR-490-5p & 1,42743639 & 0,01886813 & 0,760274481 & Up-regulated \\
\hline & hsa-miR-450b-5p & 1,11411811 & 0,02263153 & 0,763303449 & Up-regulated \\
\hline & hsa-miR-374b-5p & 0,86524386 & 0,02512358 & 0,763303449 & Up-regulated \\
\hline & hsa-miR-374c-3p & 0,87017312 & 0,02523843 & 0,763303449 & Up-regulated \\
\hline & hsa-miR-143-3p & 0,78495077 & 0,02900217 & 0,763303449 & Up-regulated \\
\hline & hsa-miR-21-3p & 0,79317052 & 0,0291757 & 0,763303449 & Up-regulated \\
\hline & hsa-miR-675-5p & 1,1546043 & 0,03213608 & 0,763303449 & Up-regulated \\
\hline & hsa-miR-671-5p & 1,76630085 & 0,03293839 & 0,763303449 & Up-regulated \\
\hline & hsa-miR-146a-5p & 0,86261284 & 0,03359957 & 0,763303449 & Up-regulated \\
\hline & hsa-miR-489-3p & 1,02467619 & 0,03372793 & 0,763303449 & Up-regulated \\
\hline & hsa-miR-96-5p & $-2,45982286$ & 0,03878464 & 0,77095716 & Down-regulated \\
\hline & hsa-miR-509-3p & 0,86545472 & 0,03909277 & 0,77095716 & Up-regulated \\
\hline & hsa-miR-190b-5p & 2,26556495 & 0,04749263 & 0,77095716 & Up-regulated \\
\hline & hsa-miR-371b-5p & $-0,92943309$ & 0,04875339 & 0,77095716 & Down-regulated \\
\hline & hsa-miR-520d-5p & $-0,6667811$ & 0,04979001 & 0,77095716 & Down-regulated \\
\hline & hsa-miR-371a-3p & $-0,92563764$ & 0,04993063 & 0,77095716 & Down-regulated \\
\hline & hsa-miR-412-5p & 0,86533956 & 0,0514002 & 0,77095716 & Up-regulated \\
\hline & hsa-miR-324-5p & $-1,01010797$ & 0,05178532 & 0,77095716 & Down-regulated \\
\hline & hsa-miR-410-3p & 0,63475365 & 0,05378966 & 0,77095716 & Up-regulated \\
\hline & hsa-miR-139-5p & 0,66901369 & 0,0543406 & 0,77095716 & Up-regulated \\
\hline \multirow[t]{7}{*}{ T. gondii (*treatment exclusive) } & hsa-miR-548l & & 0,01756271 & 0,760274481 & Up-regulated \\
\hline & hsa-miR-449a & & 0,02617767 & 0,763303449 & Up-regulated \\
\hline & hsa-miR-6125 & & 0,03454366 & 0,763303449 & Up-regulated \\
\hline & hsa-miR-4638-3p & & 0,03454366 & 0,763303449 & Up-regulated \\
\hline & hsa-miR-6860 & & 0,03454366 & 0,763303449 & Up-regulated \\
\hline & hsa-miR-4660 & & 0,03454366 & 0,763303449 & Up-regulated \\
\hline & hsa-miR-2115-5p & & 0,04354449 & 0,77095716 & Up-regulated \\
\hline
\end{tabular}

*Treatment Exclusive: refers to miRNAs sequenced only in the T. cruzi or T. gondii infected condition (treatment), they are not expressed in control explants.

proliferation, T-helper 17 cell differentiation, T-cell activation, and response to estrogen (Figure 2B).

In addition, we performed GO and KEGG analyses to identify different pathologies in which the T. cruzi- and T. gondii-induced
DEMs were related. In T. cruzi vs. control samples, significantly enriched DEMs were related to metabolic syndrome, IgAnephropathy, acute childhood lymphoblastic leukemia, atherosclerosis, oral lichen planus, human papilloma virus 


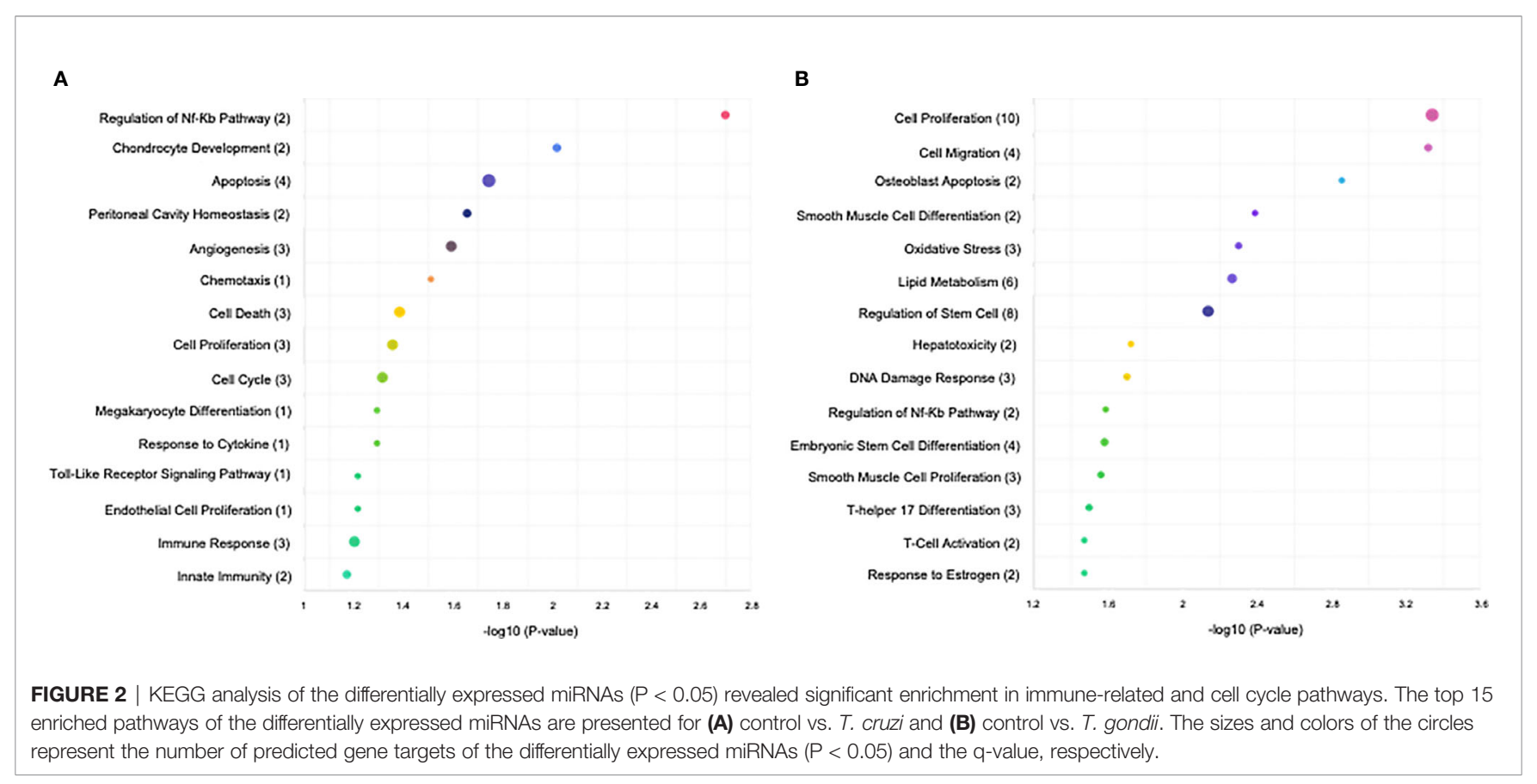

infection, psoriasis, neuropsychiatric disorders, heart diseases, pancreatic carcinoma, Löfgren's syndrome, Mycobacterium tuberculosis infection, male infertility, and gastric carcinoma (Figure 3A). In T. gondii vs. control samples, the significantly enriched DEMs were related to ankylosing spondylitis, type 2 diabetes mellitus, hypertrophic cardiomyopathy, congenital heart disease, fetal alcohol syndrome, pulmonary hypertension, ulcerative colitis, cystic fibrosis, vascular diseases, human cytomegalovirus infection, muscular dystrophy, liver diseases, coxsackievirus infection, and diabetic retinopathy (Figure 3B).
Specific GO and KEGG enrichment analyses focused on the functions of DEMs in response to both parasites that were related to parasitic diseases and/or placenta pathology. These DEMs are listed in Table 3. In this context, we found that the significantly enriched DEMs were related to the regulation of apoptosis, wound healing, cardiomyocyte apoptosis, heart development, skeletal muscle cell differentiation, Toll-like receptor signaling pathway, innate immunity, epithelial to mesenchymal transition, chromatin remodeling, and nephrotoxicity (Figure 4). Moreover, we analyzed the significantly enriched DEMs related

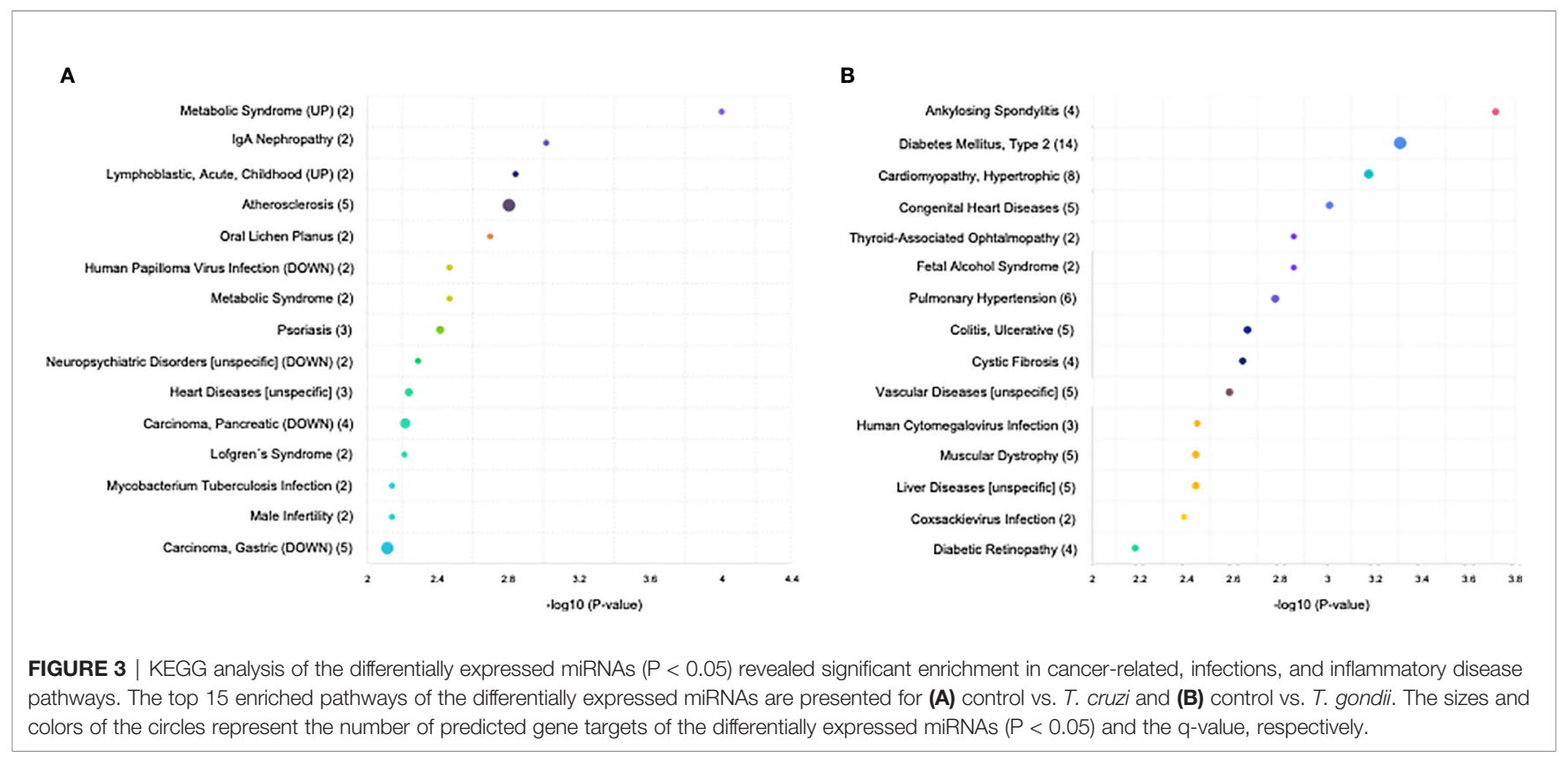


TABLE 3 | Sequenced miRNA related to placental and/or parasitic diseases in HPE challenged with $10^{5}$ T. cruzi trypomastigotes or T. gondii tachyzoites during 2 h.

\begin{tabular}{|c|c|c|c|c|}
\hline \multirow[t]{2}{*}{ miRNAs } & \multicolumn{2}{|c|}{ Control vs T. cruzi } & \multicolumn{2}{|c|}{ Control vs T. gondii } \\
\hline & Expression & p-value & Expression & p-value \\
\hline hsa-miR-3074 & Up-regulated & 0,282778175 & Down-regulated & 0,906716427 \\
\hline hsa-miR-518e-5p & Down-regulated & 0,736493282 & Up-regulated & 0,655921033 \\
\hline hsa-miR-127-3p & Down-regulated & 0,454473692 & Up-regulated & 0,899001399 \\
\hline hsa-miR-512-3p & Up-regulated & 0,893095693 & Up-regulated & 0,554888541 \\
\hline hsa-miR-516a-5p & Down-regulated & 0,573579895 & Down-regulated & 0,875664943 \\
\hline hsa-miR-376a-3p & Up-regulated & 0,26239456 & Up-regulated & 0,890873441 \\
\hline hsa-miR-523-5p & Down-regulated & 0,736493282 & Up-regulated & 0,655921033 \\
\hline hsa-miR-517-5p & Down-regulated & 0,356215385 & Down-regulated & 0,131367096 \\
\hline hsa-miR-523-3p & Up-regulated & 0,558965508 & Down-regulated & 0,070624754 \\
\hline hsa-miR-519a-5p & Down-regulated & 0,902087668 & Up-regulated & 0,683786458 \\
\hline hsa-miR-526a-5p & Down-regulated & 0,878621126 & Up-regulated & 0,975623088 \\
\hline hsa-miR-519a-3p & Up-regulated & 0,855200309 & Down-regulated & 0,216046946 \\
\hline hsa-miR-518e-3p & Up-regulated & 0,878596629 & Down-regulated & 0,062749763 \\
\hline hsa-miR-520c-5p & Down-regulated & 0,878525243 & Up-regulated & 0,981710662 \\
\hline hsa-miR-526a-3p & Down-regulated & 0,648457618 & Down-regulated & 0,194744348 \\
\hline hsa-miR-29b-3p & Up-regulated & 0,688264908 & Down-regulated & 0,461394462 \\
\hline hsa-miR-520c-3p & Down-regulated & 0,492741665 & Down-regulated & 0,321148465 \\
\hline hsa-miR-133a-3p & Up-regulated & 0,800691009 & Down-regulated & 0,140142195 \\
\hline hsa-miR-525-5p & Down-regulated & 0,892625222 & Down-regulated & 0,624806979 \\
\hline hsa-miR-525-3p & Up-regulated & 0,896175408 & Down-regulated & 0,075998808 \\
\hline hsa-miR-519c-5p & Down-regulated & 0,736493282 & Up-regulated & 0,655921033 \\
\hline hsa-miR-518b & Up-regulated & 0,991656949 & Down-regulated & 0,078353573 \\
\hline hsa-miR-519c-3p & Down-regulated & 0,262008953 & Down-regulated & 0,786169155 \\
\hline hsa-miR-520e-5p & Down-regulated & 0,485165729 & Up-regulated & 0,996288443 \\
\hline hsa-miR-520e-3p & Up-regulated & 1 & Up-regulated & 0,818473073 \\
\hline hsa-miR-21-5p & Up-regulated & 0,783933507 & Up-regulated & 0,129614377 \\
\hline hsa-miR-21-3p & Up-regulated & 0,749764521 & Up-regulated & 0,029175701 \\
\hline hsa-miR-517a-3p & Up-regulated & 0,596271375 & Down-regulated & 0,461347018 \\
\hline hsa-miR-519e-5p & Up-regulated & 0,105129034 & Up-regulated & 0,757374294 \\
\hline hsa-miR-519e-3p & Up-regulated & 0,385269175 & Up-regulated & 0,69099671 \\
\hline hsa-miR-518d-5p & Down-regulated & 0,878525243 & Up-regulated & 0,981710662 \\
\hline hsa-miR-520g-5p & Up-regulated & 0,892300156 & Up-regulated & 0,751979117 \\
\hline hsa-miR-518d-3p & Up-regulated & 0,55414416 & Down-regulated & 0,942071295 \\
\hline hsa-miR-520b-5p & Down-regulated & 0,421187791 & Down-regulated & 0,477328076 \\
\hline hsa-miR-520g-3p & Up-regulated & 0,728189158 & Down-regulated & 0,352658846 \\
\hline hsa-miR-519a-2-5p & Down-regulated & 0,421187791 & Down-regulated & 0,477328076 \\
\hline hsa-miR-520b-3p & Up-regulated & 0,530509712 & Down-regulated & 0,954450249 \\
\hline hsa-miR-517c-3p & Up-regulated & 0,760586655 & Down-regulated & 0,675509383 \\
\hline hsa-miR-524-5p & Down-regulated & 0,490615316 & Down-regulated & 0,115381834 \\
\hline hsa-miR-210-5p & Up-regulated & 0,038576256 & Up-regulated & 0,576691951 \\
\hline hsa-miR-204-5p & Up-regulated & 0,62227226 & Down-regulated & 0,583793777 \\
\hline hsa-miR-524-3p & Down-regulated & 0,907890609 & Down-regulated & 0,099098809 \\
\hline hsa-miR-519b-5p & Down-regulated & 0,736493282 & Up-regulated & 0,655921033 \\
\hline hsa-miR-210-3p & Up-regulated & 0,497217414 & Down-regulated & 0,843820092 \\
\hline hsa-miR-378a-5p & Down-regulated & 0,915728534 & Down-regulated & 0,504825517 \\
\hline hsa-miR-526b-5p & Down-regulated & 0,501400731 & Down-regulated & 0,534886926 \\
\hline hsa-miR-519b-3p & Up-regulated & 0,996536077 & Up-regulated & 0,986254335 \\
\hline hsa-miR-518a-5p & Down-regulated & 0,396658878 & Down-regulated & 0,099042516 \\
\hline hsa-miR-520d-5p & Down-regulated & 0,163923317 & Down-regulated & 0,049790011 \\
\hline hsa-miR-526b-3p & Up-regulated & 0,690372369 & Down-regulated & 0,356016985 \\
\hline hsa-miR-520d-3p & Down-regulated & 0,368956264 & Down-regulated & 0,595399092 \\
\hline hsa-miR-30e-3p & Up-regulated & 0,708643074 & Down-regulated & 0,606142557 \\
\hline hsa-miR-520h & Up-regulated & 0,420508027 & Down-regulated & 0,48103803 \\
\hline hsa-miR-519d-5p & Down-regulated & 0,699132481 & Down-regulated & 0,210250996 \\
\hline hsa-miR-515-5p & Down-regulated & 0,451420159 & Down-regulated & 0,479490879 \\
\hline hsa-miR-519d-3p & Up-regulated & 0,795099209 & Down-regulated & 0,018848296 \\
\hline hsa-miR-515-3p & Down-regulated & 0,961837732 & Down-regulated & 0,056222245 \\
\hline hsa-miR-518c-5p & Down-regulated & 0,433629597 & Down-regulated & 0,461615584 \\
\hline hsa-miR-155-5p & Up-regulated & 0,089556012 & Up-regulated & 0,307144182 \\
\hline hsa-miR-518c-3p & Up-regulated & 0,825417746 & Down-regulated & 0,393062915 \\
\hline hsa-miR-520a-5p & Up-regulated & 0,829237058 & Down-regulated & 0,786085152 \\
\hline
\end{tabular}

(Continued) 
TABLE 3 | Continued

\begin{tabular}{|c|c|c|c|c|}
\hline \multirow[t]{2}{*}{ miRNAs } & \multicolumn{2}{|c|}{ Control vs T. cruzi } & \multicolumn{2}{|c|}{ Control vs T. gondii } \\
\hline & Expression & p-value & Expression & p-value \\
\hline hsa-miR-376a-5p & Down-regulated & 0,101845272 & Down-regulated & 0,009287146 \\
\hline hsa-miR-520a-3p & Up-regulated & 0,303331111 & Down-regulated & 0,733502488 \\
\hline hsa-miR-144-5p & Up-regulated & 0,979441392 & Down-regulated & 0,781688613 \\
\hline hsa-miR-204-5p & Up-regulated & 0,62227226 & Down-regulated & 0,583793777 \\
\hline hsa-miR-424-5p & Up-regulated & 0,52797867 & Down-regulated & 0,318765339 \\
\hline hsa-miR-346 & Down-regulated & 0,996223653 & Up-regulated & 0,484560011 \\
\hline
\end{tabular}

to transcription activation and found that the following transcription factors or its activators were modulated by them: MYOG, calcineurin, AP-1, TNFSF12, NFאB1, myogenin, MYOD, MYF5, MRF4, and TP53 (Figure 5).

\section{Validation of miRNA Expression by RT- qPCR}

Validation of miRNAs associated with parasite infection or pregnancy related pathologies was performed by selecting six miRNAs [miR-3074 (26), miR-127-3p (27, 28), miR-30e-3p (29), miR-512-3p (30), miR-515-5p (31), and miR-190b (32)] for confirmation by real time PCR to verify the DEM expression levels. Expression of miR-3074, miR-127-3p, and miR-30e-3p (Figures 6A-C) was analyzed in HPE in response to both parasites. miR-512-3p and miR-515-5p (Figures 6D-E) expression was determined in response to T. cruzi infection and miR-190b expression in response to T. gondii infection (Figure 6F). All selected miRNAs except for miR-30e-3p (Figure 6C) were differentially expressed. Thus, miR-3074 expression (Figure 6A) was significantly decreased (T. cruzi: $57.03 \pm 19.99 \%, \mathrm{p} \leq$ 0.01 ; T. gondii: $69.84 \pm 24.67 \%, \mathrm{p} \leq 0.01)$ with respect to the control but not the infected samples. Decreased miR-3074 expression was expected in the T. gondii infected samples but not in the T. cruzi infected samples. According to the miRNA Seq data, miR-3074 was upregulated in T. cruzi challenged samples. Similar results were observed for miR-127-3p (Figigure 6B). Expression of miR-127-3p was significantly decreased in HPE infected with either parasite (T. cruzi: $68.218 \pm 16.41 \%, \mathrm{p} \leq 0.01$; T. gondii: $73.13 \pm 22.45 \%, \mathrm{p} \leq 0.01)$ compared to the control but not to infected samples; we expected an increase in miR-127-3p expression in the presence of $T$. gondii since in the miRNA Seq data this particular miRNA was increased (Table 3). RT-qPCR validation results for miR-512-3p, miR-515-5p, and miR-190b confirmed the miRNA Seq data. Thus, miR-512-3p expression increased (40.83 $\pm 22.53 \%, \mathrm{p} \leq 0.01)$ (Figure 6D) and miR-515-5p expression decreased $(21.44 \pm 8.60 \%, \mathrm{p} \leq 0.01)$ (Figure 6E) significantly in T. cruzi infected samples. In T. gondii infected HPE, miR-190b expression was significantly increased (59.02 \pm $37.73 \%, \mathrm{p} \leq 0.01$ ) (Figure 6F).

\section{DISCUSSION}

Pathogens have evolved strategies to exploit resources from their hosts to maximize their own survival, replication, and dissemination. Thus, different kinds of pathogens (including

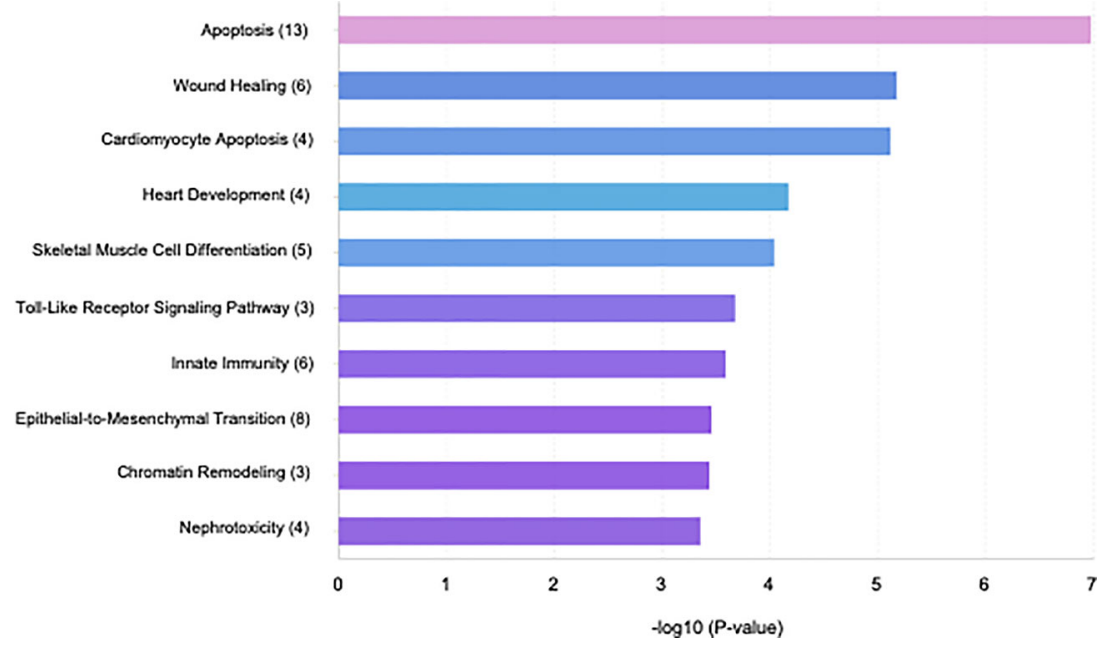

FIGURE 4 | Bar plot illustrating the top 10 significant miRNA-function associations of the sequenced miRNA related to placental and/or parasitic diseases in human placental explants infected with T. cruzi or T. gondii. 


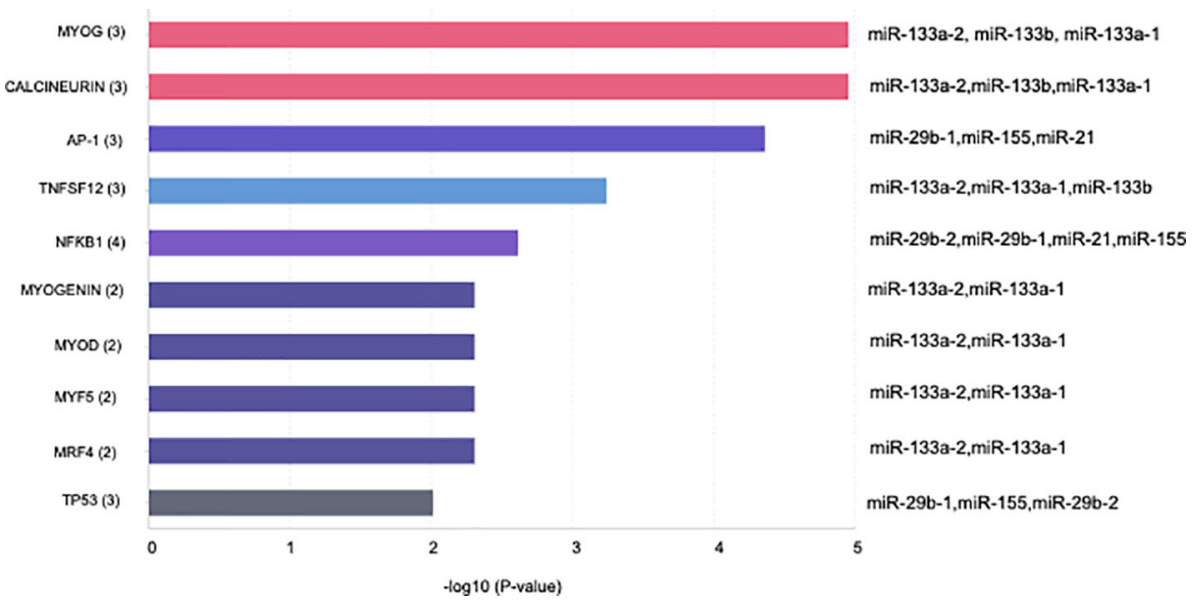

FIGURE 5 | Bar plot illustrating the top 10 significant miRNA transcription factor associations of the sequenced miRNA related to placental and/or parasitic diseases in human placental explants infected with $T$. cruzi or $T$. gondii.

A

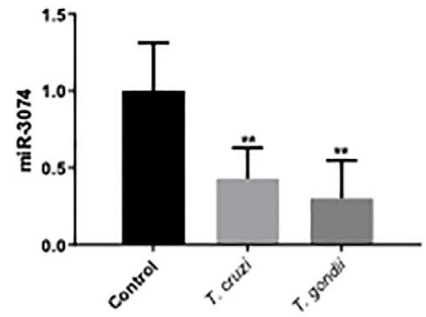

D

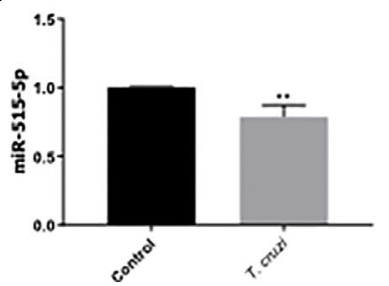

B

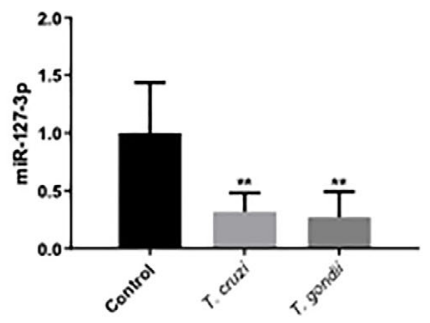

E

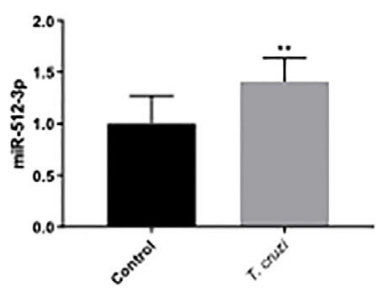

C

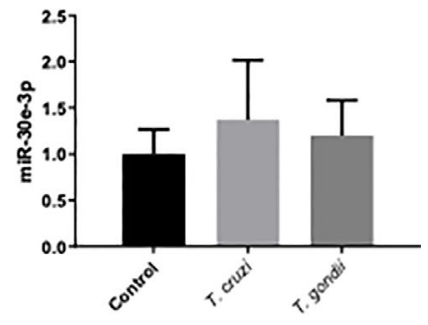

$\mathbf{F}$

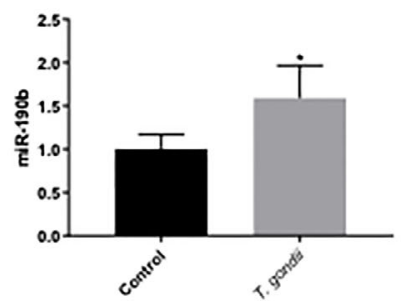

FIGURE 6 | Validation of miRNAs related to placental and/or parasitic diseases using RT-qPCR. Human placental explants were challenged with $10^{5}$ T. cruzi trypomastigotes or T. gondii tachyzoites for $2 \mathrm{~h}$. The presence of miRNA was determined by real-time PCR. T. cruzi and T. gondii decreased miR-3074 and miR127-3p expression, while no change was observed in miR-30e-3p expression (A-C). T. cruzi inhibited miR-515-5p expression and induced miR512-3p (D, E). T. gondii induced miR-190b-5p (F). All values are the mean \pm S.D. and correspond to at least three independent experiments that were performed in triplicate. Data were normalized in terms of the control values and analyzed by Student's t-test or ANOVA. ${ }^{*} \mathrm{p} \leq 0.05 ;{ }^{* *} \mathrm{p} \leq 0.01$.

parasites) have developed sophisticated mechanisms that include hijacking host cellular machinery to modulate host gene expression to inhibit defense responses $(16,32,33)$. Both of the parasites studied here are able to manipulate host gene expression. For instance, during $T$. cruzi cell and tissue invasion, cell reprogramming affects cellular stress responses, host metabolism, and a significant number of transcription factors $(16,34)$. T. gondii also reprograms host cells, primarily targeting cell-specific transcription factors that regulate host defenses (i.e., NF- $\kappa B$, interferon regulatory factor, and JAK/ STAT) by regulating their intrinsic activities and expression levels (35). In addition, certain parasites including T. cruzi and T. gondii can alter host miRNA expression to favor both parasite clearance and infection $(18,19)$. Moreover, different strains of $T$. gondii can induce specific miRNAs in mice that have been proposed as biomarkers for early infection $(19,36)$.

Mature miRNAs regulate the expression of over $30 \%$ of fundamental genes; these are involved in key biological 
processes including development, cellular proliferation and differentiation, apoptosis, metabolism, and immune response $(18,19,37)$; all of these determine infection success or failure. Moreover, epigenetic and genetic defects in miRNAs and their processing machinery are a common hallmark of infection and diseases that include pregnancy-specific pathologies such as preeclampsia $(17,38)$.

Most of the transcriptomic studies as well as those analyzing miRNA profiles have focused on a single type of cell response (34) or on tissues or organs in animal models $(39,40)$; no studies have focused on human tissues. The present study is the first report on the miRNA profile of the human placenta in response to T. cruzi or T. gondii infection. We identified 680 and 686 DEMs, respectively, in T. cruzi and T. gondii infected samples. T. cruzi modulated only 14 DEMs with a minimum of a 1.5 -fold change in expression and a $95 \%$ probability of being differentially expressed (Figure 1A). In contrast, the number of DEMs increased to 42 in T. gondii challenged HPE (Figure 2B). Our results showed that the DEMs identified here are related to the regulation of different fundamental cellular processes (Figure 2) as well as different pathologies (Figure 3). Moreover, fundamental cellular processes related to placenta pathologies and embryonic development are affected by the presence of both parasites (Figures 4-5). It is important to point out, that tissue response to infection is relevant during disease progression. The presence of the parasites leads to tissue damage as well as immune and regulatory/repair responses, which can lead to fibrosis and tissue dysfunction as observed in chagasic cardiomyopathy (41) or Toxoplasma induced encephalitis in immune-compromised individuals (42).

Three miRNAs, miR-21, miR-146a/b, and miR-210, were overrepresented in most of the ontology terms (Table 2, Supplementary Tables 3-4). Previous studies have implicated these miRNAs in immune and inflammatory response regulation via macrophage polarization controlled through transcription factor regulation in response to signals from the microenvironment $(43,44)$. Concordantly, in T. cruzi-infected mice, increased miR-21 expression in the heart has been correlated with a parasitemia peak at 30 days post-infection (39). In placenta, miR-21 has been associated with trophoblast differentiation and invasion and miR-21 dysregulation leads to placental pathology (45). MiR-146a is a negative feedback regulator in TLR-4 signaling that acts by repressing TRAF6 to inhibit $\mathrm{NF \kappa B}$ transcription factor activation $(46,47)$. In macrophages, TRAF6 mediates the induction of the proinflammatory cytokine IL-12, which is essential to control $T$. gondii infection (48). TRAF6 activation is also required for vacuole-lysosome fusion, a fundamental step during T. gondii infection (49). Our results showed that in HPEs, T. gondii and T. cruzi infection increased miR-146a expression. Our previous studies showed that both parasites modulate placental immune response differentially through TLRs and NFKB pathways in HPEs (9, 10) Interestingly, the inhibition of these pathways increased the DNA loads of both parasites in HPEs (10). Increased $T$. gondii infection in placental tissue is also induced by TLR-4 inhibition (9). In addition, increased levels of miR- 146a have been reported in the brains of mice infected with $T$. gondii, moreover, miR-146a ablation affects early parasite burden and improves survival (50). It was previously reported that miR210 is induced by damage associated molecular patterns (51). In preeclamptic placentas, miR-210 is increased (52); in the present study, miR-210 was increased in HPE infected with T. cruzi but not with T. gondii. Expression of miR-210 can be directly regulated by the specific binding of NF- $\kappa \mathrm{B}$ p50 to its putative promoter (53). In this context, it is important to mention that $T$. cruzi, but not T. gondii, infection of HPE activates both NF- $\mathrm{BB}$ signaling pathways (10). Therefore, the increased level of miR210 might be a placental response to signal transduction pathway activation.

In addition, several identified pathways, important, e.g. for chondrocyte development, megakaryocyte smooth and muscle cell differentiation, hepatotoxicity, and DNA damage response, are neither related to infection or with placental tissues (Figure 3). This can be explained be the fact, that miRNAs target multiple genes, while individual genes are targeted by multiple miRNAs. Moreover, the same miRNA regulates different genes in different tissues and organs $(54,55)$. Here, we chose to validate six miRNAs that were associated specifically with parasite infection and/or pregnancy related pathologies (Figure 6). Deregulation of $\mathrm{miR}-30 \mathrm{e}-3 \mathrm{p}$ has been reported in mice that were experimentally infected with $T$. gondii (40). This miRNA is also related to Chagas cardiomyopathy (39) and is upregulated in placentas with intrauterine growth restriction (56). Nonetheless, miR-30e-3p expression was unaffected by $T$. cruzi or T. gondii infection in HPE (Figure 6C). Increased miR3074-5p expression has been described in placentas from recurrent miscarriages (57) and in livers from T. gondiiinfected cats (42). However, miR-3074-5p expression was diminished in HPE infected either with T. cruzi or T. gondii (Figure 6A). The differences between our results and the reported data might be explained by differences in the studied organs (heart and liver versus placenta) and the complexity of the above mentioned placental pathologies. MiR-127 is a placenta-specific miRNA codified in the C14MC cluster (58) and its levels are decreased in placenta-related pathologies such as recurrent miscarriage and small-for-gestational age $(59,60)$; the downregulation of MiR-127 was also detected in babies infected congenitally with either parasite $(3,4)$. Concordantly, our results showed that HPE infection with either T. cruzi or T. gondii led to the decrease of this miRNA (Figure 6B). Moreover, a decreased expression of miR-127-3p in non-placental tissues has been reported during $T$. gondii infection in mice and cats $(40,42,61)$, but there is no report regarding miR-127-3p expression in response to T. cruzi infection. Both miR-515-5p and $\mathrm{miR}-512-3 \mathrm{p}$ are placenta-specific miRNAs that are codified in the C19MC cluster (62). Decreased miR-515-5p expression is related to fetal growth restriction (63) and preeclampsia (64). Importantly, this miRNA inhibits human trophoblast differentiation by directly repressing the aromatase P450 (CYP19A1), frizzled 5 (FZD5), and glial cells missing 1 transcription factor (GCM1) genes (65). Trophoblast differentiation is part of the trophoblast epithelial turnover 


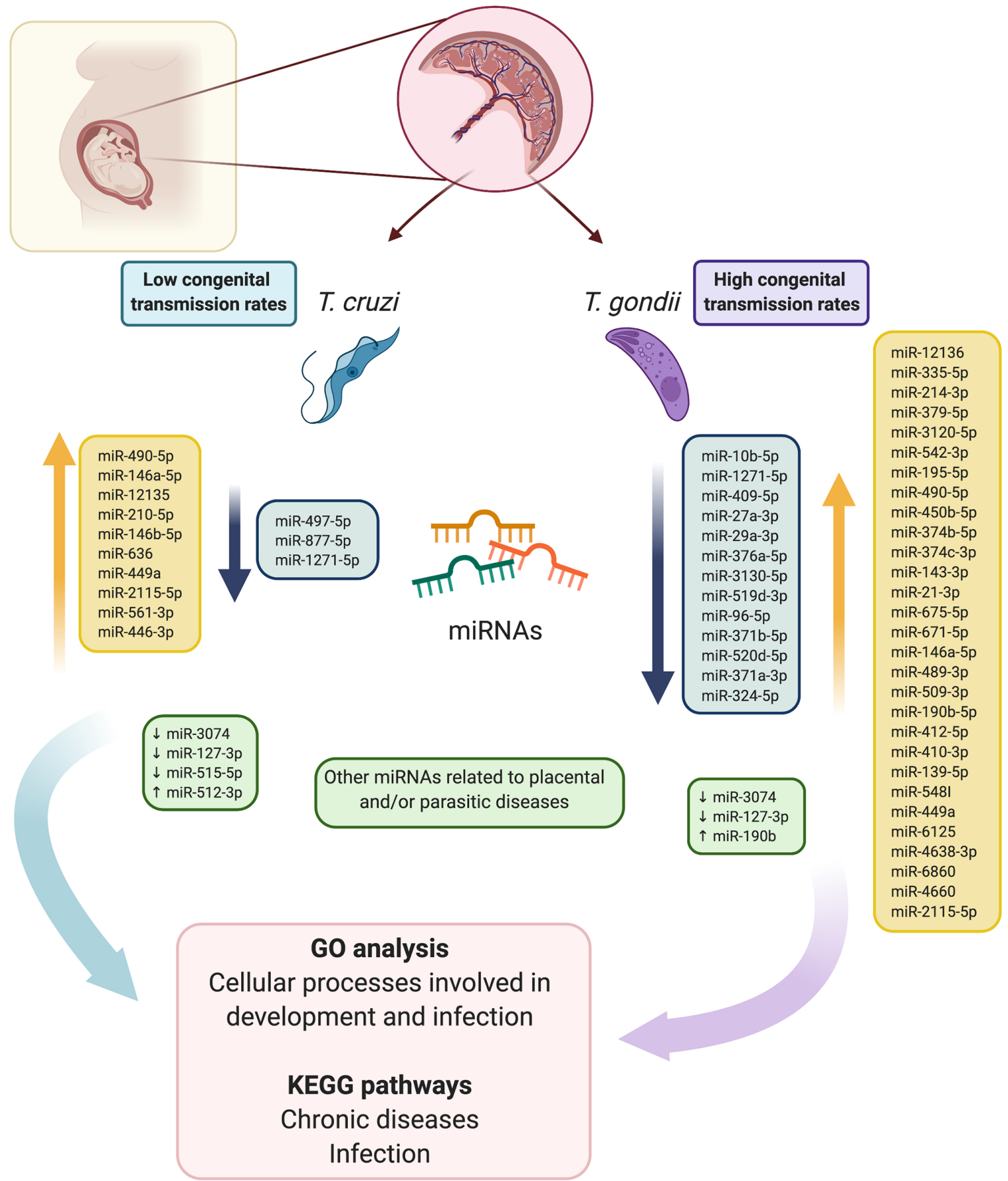

FIGURE 7 | Schematic summary of the comparative analysis of RNA sequencing-based miRNA profiles in HPEs in response to ex vivo T. cruzi and T. gondii infection. Fourteen, and 42 DEMs were identified in T. cruzi and T. gondii infected samples. In T. cruzi infected HPEs, 5 miRNAs were downregulated and 9 were upregulated. In $T$. gondii infected HPEs, 13 miRNAs were downregulated and 29 were upregulated. In addition, five miRNAs that are associated specifically with parasite infection and/or pregnancy-related pathologies were validated. GO analysis revealed that the predicted targets of the DEMs were different cellular processes involved in development and immunity, and most of the identified KEGG pathways were related to chronic diseases and infection. Considering that the DEMs identified herein modulate crucial host cellular targets potentially determining the success of infection, these miRNAs might explain the differences in the congenital transmission rates of the two parasites. This figure was created using BioRender.com. 
and it has been proposed that this mechanism is part of an antiparasitic placental response against $T$. cruzi infection (11, $13,66,67)$. Therefore, our reported decrease of miR-515-5p expression during ex vivo T. cruzi infection of HPE (Figure 6D) might be at least partially responsible for the parasite-induced trophoblast differentiation. In contrast, miR-512-3p was upregulated in HPE in response to T. cruzi infection (Figure 6E). Interestingly, miR-512-3p confers resistance to vesicular stomatitis virus in non-placental recipient cells (68) and represses the caspase 8 inhibitor c-FLIP (cellular FLICE-like inhibitory protein); it consequently increases caspase 8 activity (69). Caspase 8 regulates trophoblast differentiation and apoptotic cell death and is activated by T. cruzi (66). Therefore, miR-512-3p upregulation might also be a protective placental response to $T$. cruzi infection, as it is to viral infection. The upregulation of miR-190b in HPE during $T$. gondii infection was observed in the RNAseq analysis (Figure 1B, Table 3), then validated by qPCR (Figure 6F). Upregulation of miR-190b promotes cell proliferation and migration and reduces cell apoptosis in different types of cancer $(70,71)$. Parasites modulate apoptotic responses in infected cells to avoid rapid clearance; $T$. gondii is particularly capable of blocking apoptosis by different mechanisms (33). In neurons, increased miR-190b expression also increases cell viability, suppresses autophagy, and significantly decreases the levels of pro-inflammatory TNF- $\alpha$, IL- 6 , and IL- $1 \beta$ cytokines (72). In this context, we have shown that $T$. gondii, in contrast to $T$. cruzi, does not induce pro-inflammatory cytokines in HPE (9). Therefore, it is postulated that the lack of pro-inflammatory cytokine secretion in response to T. gondii in HPE might be related to an increase in $\mathrm{miR}-190 \mathrm{~b}$ expression and that, together with the modulation of the apoptotic pathway, it could allow parasite persistence and infection in the placenta.

In conclusion, the present study provides a comparative analysis of RNA sequencing-based miRNA profiles in HPE in response to ex vivo T. cruzi and T. gondii infection (Figure 7). Our findings provide new insight into the capacity of both parasites to modulate host gene expression. GO analysis revealed that the predicted targets of the DEMs were different cellular processes involved in development and immunity, and most of the identified KEGG pathways were related to chronic diseases and infection. Considering that the DEMs identified herein modulate crucial host cellular targets that participate in determining the success of infection, these miRNAs might explain the differences in congenital transmission rates. Molecules of the different pathways that are regulated by miRNAs and modulated during infection, as well as the miRNAs themselves, may be potential targets for the therapeutic control of either congenital Chagas disease or toxoplasmosis.

\section{REFERENCES}

1. Dotters-Katz S, Kuller J, Heine RP. Parasitic infections in pregnancy. Obstet Gynecol Surv (2011) 66:515-25. doi: 10.1097/OGX.0b013e3182385fde

2. Błaszkowska J, Góralska K. Parasites and fungi as a threat for prenatal and postnatal human development. Ann Parasitol (2014) 60:225-34.

\section{DATA AVAILABILITY STATEMENT}

The RNA-seq data reported in the present study have been submitted to the NCBI SRA database (http:// www.ncbi.nlm.nih.gov/bioproject/656620; accession number PRJNA656620). All other data supporting the findings can be found in the article/Supplementary Material.

\section{ETHICS STATEMENT}

The studies involving human participants were reviewed and approved by Ethical Committee of the "Servicio de Salud Metropolitano Norte" Santiago de Chile, Chile. The patients/ participants provided their written informed consent to participate in this study.

\section{AUTHOR CONTRIBUTIONS}

LM and UK conceived of and planned experiments. LM, CC, MR-P, AL, and JG-M carried out experiments. JM and HP contributed to the interpretation of the results. UK and LM wrote the manuscript. All authors contributed to the article and approved the submitted version.

\section{FUNDING}

UK received a grant from the Network of the European Union, Latin America and the Caribbean Countries on Joint Innovation and Research Activities (ERANet-LAC; grant number ERANet17/HLH-0142). UK, JM, and CC received grants from the National Fund for Scientific and Technological Development (FONDECYT; grant numbers 1190341, 1170126, and 3180452, respectively).

\section{ACKNOWLEDGEMENTS}

The authors thank Dr. Sergio Angel for providing the Toxoplasma gondii parasites.

\section{SUPPLEMENTARY MATERIAL}

The Supplementary Material for this article can be found online at: https://www.frontiersin.org/articles/10.3389/fimmu.2020. 595250/full\#supplementary-material

3. Carlier Y, Truyens C, Deloron P, Peyron F. Congenital parasitic infections: a review. Acta Trop (2012) 121:55-70. doi: 10.1016/j.actatropica.2011.10.018

4. Kemmerling U, Osuna A, Schijman AG, Truyens C. Congenital Transmission of Trypanosoma cruzi: A Review About the Interactions Between the Parasite, the Placenta, the Maternal and the Fetal/Neonatal Immune Responses. Front Microbiol (2019) 10:1854. doi: 10.3389/fmicb.2019.01854 
5. Schmuñis G. Status of and cost of Chagas disease worldwide. Lancet Infect Dis (2013) 13:283-4. doi: 10.1016/S1473-3099(13)70032-X

6. Pérez-Molina JA, Molina I. Chagas disease. Lancet (London England) (2018) 391:82-94. doi: 10.1016/S0140-6736(17)31612-4

7. Melo MB, Jensen KDC, Saeij JPJ. Toxoplasma gondii effectors are master regulators of the inflammatory response. Trends Parasitol (2011) 27:487-95. doi: 10.1016/j.pt.2011.08.001

8. Rendell VR, Gilman RH, Valencia E, Galdos-Cardenas G, Verastegui M, Sanchez $\mathrm{L}$, et al. Trypanosoma cruzi-infected pregnant women without vector exposure have higher parasitemia levels: implications for congenital transmission risk. PloS One (2015) 10:e0119527. doi: 10.1371/journal.pone.0119527

9. Castillo C, Muñoz L, Carrillo I, Liempi A, Gallardo C, Galanti N, et al. Ex vivo infection of human placental chorionic villi explants with Trypanosoma cruzi and Toxoplasma gondii induces different Toll-like receptor expression and cytokine/chemokine profiles. Am J Reprod Immunol (2017) 78:1-8. doi: $10.1111 /$ aji. 12660

10. Liempi A, Castillo C, Medina L, Rojas M, Maya JD, Parraguez VH, et al. Ex vivo infection of human placental explants with Trypanosoma cruzi and Toxoplasma gondii: Differential activation of NF kappa B signaling pathways. Acta Trop (2019) 199:105153. doi: 10.1016/j.actatropica.2019.105153

11. Castillo C, Muñoz L, Carrillo I, Liempi A, Medina L, Galanti N, et al. Toll-like receptor-2 mediates local innate immune response against Trypanosoma cruzi in ex vivo infected human placental chorionic villi explants. Placenta (2017) 60:40-6. doi: 10.1016/j.placenta.2017.10.005

12. Sen R, Nayak L, De RK. A review on host-pathogen interactions: classification and prediction. Eur J Clin Microbiol Infect Dis (2016) 35:1581-99. doi: 10.1007/s10096-016-2716-7

13. Liempi A, Castillo C, Carrillo I, Muñoz L, Droguett D, Galanti N, et al. A local innate immune response against Trypanosoma cruzi in the human placenta: The epithelial turnover of the trophoblast. Microb Pathog (2016) 99:123-9. doi: $10.1016 /$ j.micpath.2016.08.022

14. De Souza W, Ulisses de Carvalho TM. Active penetration of Trypanosoma cruzi into host cells: Historical considerations and current concepts. Front Immunol (2013) 4:2. doi: 10.3389/fimmu.2013.00002

15. Hakimi Ma, Cannella D. Apicomplexan parasites and subversion of the host cell microRNA pathway. Trends Parasitol (2011) 27:481-6. doi: 10.1016/ j.pt.2011.07.001

16. Castillo C, Carrillo I, Libisch G, Juiz N, Schijman AG, Robello C, et al. Hostparasite interaction: Changes in human placental gene expression induced by Trypanosoma cruzi. Parasit Vectors (2018) 11:1-13. doi: 10.1186/s13071-0182988-0

17. Hayder H, O’Brien J, Nadeem U, Peng C. MicroRNAs: crucial regulators of placental development. Reproduction (2018) 155:R259-71. doi: 10.1530/REP17-0603

18. Zheng Y, Cai X, Bradley JE. MicroRNAs in parasites and parasite infection. RNA Biol (2013) 10:377-85. doi: 10.4161/rna.23716

19. Acuña SM, Floeter-Winter LM, Muxel SM. MicroRNAs: Biological Regulators in Pathogen-Host Interactions. Cells (2020) 9:113. doi: 10.3390/cells9010113

20. Ferreira LRP, Frade AF, Santos RHB, Teixeira PC, Baron MA, Navarro IC, et al. MicroRNAs miR-1, miR-133a, miR-133b, miR-208a and miR-208b are dysregulated in Chronic Chagas disease Cardiomyopathy. Int J Cardiol (2014) 175:409-17. doi: 10.1016/j.ijcard.2014.05.019

21. Cai Y, Shen J. Modulation of host immune responses to Toxoplasma gondii by microRNAs. Parasite Immunol (2017) 39:1-6. doi: 10.1111/pim.12417

22. Sadovsky Y, Mouillet JF, Ouyang Y, Bayer A, Coyne CB. The function of trophomirs and other micrornas in the human placenta. Cold Spring Harb Perspect Med (2015) 5:1-16. doi: 10.1101/cshperspect.a023036

23. Dumont TMF, Mouillet JF, Bayer A, Gardner CL, Klimstra WB, Wolf DG, et al. The expression level of C19MC miRNAs in early pregnancy and in response to viral infection. Placenta (2017) 53:23-9. doi: 10.1016/ j.placenta.2017.03.011

24. Delorme-Axford E, Sadovsky Y, Coyne CB. The Placenta as a Barrier to Viral Infections. Annu Rev Virol (2014) 1:133-46. doi: 10.1146/annurev-virology031413-085524

25. Nardelli SC, Che F-Y, Silmon de Monerri NC, Xiao H, Nieves E, MadridAliste $\mathrm{C}$, et al. The histone code of Toxoplasma gondii comprises conserved and unique posttranslational modifications. MBio (2013) 4:e00922-13. doi: 10.1128/mBio.00922-13
26. Liempi A, Castillo C, Medina L, Galanti N, Maya JD, Parraguez VH, et al. Comparative ex vivo infection with Trypanosoma cruzi and Toxoplasma gondii of human, canine and ovine placenta: Analysis of tissue damage and infection efficiency. Parasitol Int (2020) 76:102065. doi: 10.1016/ j.parint.2020.102065

27. Lindgreen S. AdapterRemoval: Easy cleaning of next-generation sequencing reads. BMC Res Notes (2012) 5:337. doi: 10.1186/1756-0500-5-337

28. Dobin A, Davis CA, Schlesinger F, Drenkow J, Zaleski C, Jha S, et al. STAR: Ultrafast universal RNA-seq aligner. Bioinformatics (2013) 29:15-21. doi: 10.1093/bioinformatics/bts635

29. Anders S, Pyl PT, Huber W. HTSeq-A Python framework to work with highthroughput sequencing data. Bioinformatics (2015) 31:166-9. doi: 10.1093/ bioinformatics/btu638

30. Robinson MD, McCarthy DJ, Smyth GK. edgeR: A Bioconductor package for differential expression analysis of digital gene expression data. Bioinformatics (2009) 26:139-40. doi: 10.1093/bioinformatics/btp616

31. Androvic P, Valihrach L, Elling J, Sjoback R, Kubista M. Two-tailed RT-qPCR: a novel method for highly accurate miRNA quantification. Nucleic Acids Res (2017) 45:e144. doi: 10.1093/nar/gkx588

32. Villares M, Berthelet J, Weitzman JB. The clever strategies used by intracellular parasites to hijack host gene expression. Semin Immunopathol (2020) 42:215-26. doi: 10.1007/s00281-020-00779-z

33. Mammari N, Halabi MA, Yaacoub S, Chlala H, Dardé M-L, Courtioux B. Toxoplasma gondii Modulates the Host Cell Responses: An Overview of Apoptosis Pathways. BioMed Res Int (2019), 2019:6152489. doi: 10.1155/ 2019/6152489

34. Chiribao ML, Libisch G, Parodi-Talice A, Robello C. Early Trypanosoma cruzi infection reprograms human epithelial cells. BioMed Res Int (2014) 2014:439501. doi: 10.1155/2014/439501

35. Hakimi MA, Olias P, Sibley LD. Toxoplasma effectors targeting host signaling and transcription. Clin Microbiol Rev (2017) 30:615-44. doi: 10.1128/ CMR.00005-17

36. Jia B, Chang Z, Wei X, Lu H, Yin J, Jiang N, et al. Plasma microRNAs are promising novel biomarkers for the early detection of Toxoplasma gondii infection. Parasit Vectors (2014) 7:433. doi: 10.1186/1756-3305-7-433

37. Salehi M, Sharifi M. Exosomal miRNAs as novel cancer biomarkers: Challenges and opportunities. J Cell Physiol (2018) 233:6370-80. doi: $10.1002 /$ jcp. 26481

38. Mouillet JF, Ouyang Y, Coyne CB, Sadovsky Y. MicroRNAs in placental health and disease. Am J Obstet Gynecol (2015) 213:S163-72. doi: 10.1016/ j.ajog.2015.05.057

39. Navarro IC, Ferreira FM, Nakaya HI, Baron MA, Vilar-Pereira G, Pereira IR, et al. MicroRNA transcriptome profiling in heart of trypanosoma cruziinfected mice: Parasitological and cardiological Outcomes. PloS Negl Trop Dis (2015) 9:1-17. doi: 10.1371/journal.pntd.0003828

40. Hu RS, He JJ, Elsheikha HM, Zhang FK, Zou Y, Zhao GH, et al. Differential brain microRNA expression profiles after acute and chronic infection of mice with toxoplasma gondii oocysts. Front Microbiol (2018) 9:2316. doi: 10.3389/ fmicb.2018.02316

41. Machado FS, Dutra WO, Esper L, Gollob KJ, Teixeira MM, Factor SM, et al. Current understanding of immunity to Trypanosoma cruzi infection and pathogenesis of Chagas disease. Semin Immunopathol (2012) 34:753-70. doi: 10.1007/s00281-012-0351-7

42. Cong W, Zhang XX, He JJ, Li FC, Elsheikha HM, Zhu XQ. Global miRNA expression profiling of domestic cat livers following acute Toxoplasma gondii infection. Oncotarget (2017) 8:25599-611. doi: 10.18632/oncotarget.16108

43. Cătană CS, Calin GA, Berindan-Neagoe I. Inflamma-miRs in aging and breast cancer: Are they reliable players? Front Med (Lausanne) (2015) 2:85. doi: $10.3389 /$ fmed.2015.00085

44. Li H, Jiang T, Li MQ, Zheng XL, Zhao GJ. Transcriptional regulation of macrophages polarization by microRNAs. Front Immunol (2018) 9:1175. doi: 10.3389/fimmu.2018.01175

45. Chaiwangyen W, Ospina-Prieto S, Photini SM, Schleussner E, Markert UR, Morales-Prieto DM. Dissimilar microRNA-21 functions and targets in trophoblastic cell lines of different origin. Int J Biochem Cell Biol (2015) 68:187-96. doi: 10.1016/j.biocel.2015.08.018

46. Hou J, Wang P, Lin L, Liu X, Ma F, An H, et al. MicroRNA-146a Feedback Inhibits RIG-I-Dependent Type I IFN Production in Macrophages by 
Targeting TRAF6, IRAK1, and IRAK2. J Immunol (2009) 183:2150-8. doi: 10.4049/jimmunol.0900707

47. Taganov KD, Boldin MP, Chang KJ, Baltimore D. NF-KB-dependent induction of microRNA miR-146, an inhibitor targeted to signaling proteins of innate immune responses. Proc Natl Acad Sci U S A (2006) 103:12481-6. doi: 10.1073/pnas.0605298103

48. Mason NJ, Fiore J, Kobayashi T, Masek KS, Choi Y, Hunter CA. TRAF6dependent mitogen-activated protein kinase activation differentially regulates the production of interleukin- 12 by macrophages in response to Toxoplasma gondii. Infect Immun (2004) 72:5662-7. doi: 10.1128/IAI.72.10.56625667.2004

49. Subauste CS, Andrade RM, Wessendarp M. CD40-TRAF6 and autophagydependent anti-microbial activity in macrophages. Autophagy (2007) 3:245-8. doi: 10.4161/auto.3717

50. Cannella D, Brenier-Pinchart MP, Braun L, vanRooyen JM, Bougdour A, Bastien O, et al. MiR-146a and miR-155 delineate a microRNA fingerprint associated with toxoplasma persistence in the host brain. Cell Rep (2014) 6:928-37. doi: 10.1016/j.celrep.2014.02.002

51. Unlu S, Tang S, Wang E, Martinez I, Tang D, Bianchi ME, et al. Damage associated molecular pattern molecule-induced micrornas (DAMPmiRs) in human peripheral blood mononuclear cells. PloS One (2012) 7(6):e38899. doi: 10.1371/journal.pone.0038899

52. Zhu Xm, Han T, Sargent IL, Yin G, Yao Yq. Differential expression profile of microRNAs in human placentas from preeclamptic pregnancies vs normal pregnancies. Am J Obstet Gynecol (2009) 7(6):e38899. doi: 10.1016/ j.ajog.2008.12.045

53. Zhang Y, Fei M, Xue G, Zhou Q, Jia Y, Li L, et al. Elevated levels of hypoxiainducible microRNA-210 in pre-eclampsia: New insights into molecular mechanisms for the disease. J Cell Mol Med (2012) 16:249-59. doi: 10.1111/ j.1582-4934.2011.01291.x

54. Peter ME. Targeting of mRNAs by multiple miRNAs: The next step. Oncogene (2010) 29:2161-4. doi: 10.1038/onc.2010.59

55. Cai Y, Yu X, Hu S, Yu J. A Brief Review on the Mechanisms of miRNA Regulation. Genomics Proteomics Bioinformatics (2009) 7:147-54. doi: 10.1016/S1672-0229(08)60044-3

56. Wen H, Chen L, He J, Lin J. MicroRNA expression profiles and networks in placentas complicated with selective intrauterine growth restriction. Mol Med Rep (2017) 16:6650-73. doi: 10.3892/mmr.2017.7462

57. Gu Y, Zhang X, Yang Q, Wang J, He Y, Sun Z, et al. Aberrant Placental Villus Expression of miR-486-3p and miR-3074-5p in Recurrent Miscarriage Patients and Uterine Expression of These MicroRNAs during Early Pregnancy in Mice. Gynecol Obstet Invest (2016) 81:112-7. doi: 10.1159/000435879

58. Williams Z, Ben-Dov IZ, Elias R, Mihailovic A, Brown M, Rosenwaks Z, et al. Comprehensive profiling of circulating microRNA via small RNA sequencing of cDNA libraries reveals biomarker potential and limitations. Proc Natl Acad Sci U S A (2013) 110:4255-60. doi: 10.1073/pnas.1214046110

59. Yang Q, Gu WW, Gu Y, Yan NN, Mao YY, Zhen XX, et al. Association of the peripheral blood levels of circulating microRNAs with both recurrent miscarriage and the outcomes of embryo transfer in an in vitro fertilization process. J Transl Med (2018) 16(1):186. doi: 10.1186/s12967-018-1556-x

60. Rodosthenous RS, Burris HH, Sanders AP, Just AC, Dereix AE, Svensson K, et al. Second trimester extracellular microRNAs in maternal blood and fetal growth: An exploratory study. Epigenetics (2017) 12:804-10. doi: 10.1080/ 15592294.2017.1358345
61. He JJ, Ma J, Wang JL, Xu MJ, Zhu XQ. Analysis of miRNA expression profiling in mouse spleen affected by acute Toxoplasma gondii infection. Infect Genet Evol (2016) 37:137-42. doi: 10.1016/j.meegid.2015.11.005

62. Donker RB, Mouillet JF, Chu T, Hubel CA, Stolz DB, Morelli AE, et al. The expression profile of C19MC microRNAs in primary human trophoblast cells and exosomes. Mol Hum Reprod (2012) 18:417-24. doi: 10.1093/molehr/ gas 013

63. Higashijima A, Miura K, Mishima H, Kinoshita A, Jo O, Abe S, et al. Characterization of placenta-specific microRNAs in fetal growth restriction pregnancy. Prenat Diagn (2013) 33:214-22. doi: 10.1002/pd.4045

64. Hromadnikova I, Kotlabova K, Ondrackova M, Pirkova P, Kestlerova A, Novotna $\mathrm{V}$, et al. Expression profile of C19MC microRNAs in placental tissue in pregnancy-related complications. DNA Cell Biol (2015) 34:437-57. doi: $10.1089 /$ dna.2014.2687

65. Zhang M, Muralimanoharan S, Wortman AC, Mendelson CR. Primatespecific miR-515 family members inhibit key genes in human trophoblast differentiation and are upregulated in preeclampsia. Proc Natl Acad Sci (2016) 113:E7069-76. doi: 10.1073/pnas.1607849113

66. Carrillo I, Droguett D, Castillo C, Liempi A, Muñoz L, Maya JDJDJD, et al. Caspase- 8 activity is part of the BeWo trophoblast cell defense mechanisms against Trypanosoma cruzi infection. Exp Parasitol (2016) 168:9-15. doi: 10.1016/j.exppara.2016.06.008

67. Liempi A, Castillo C, Duaso J, Droguett D, Sandoval A, Barahona K, et al. Trypanosoma cruzi induces trophoblast differentiation: A potential local antiparasitic mechanism of the human placenta? Placenta (2014) 35:103542. doi: 10.1016/j.placenta.2014.09.017

68. Delorme-Axford E, Donker RB, Mouillet J-F, Chu T, Bayer A, Ouyang Y, et al. Human placental trophoblasts confer viral resistance to recipient cells. Proc Natl Acad Sci (2013) 110:12048-53. doi: 10.1073/pnas.1304718110

69. Chen F, Zhu H-H, Zhou L-F, Wu S-S, Wang J, Chen Z. Inhibition of c-FLIP expression by miR-512-3p contributes to taxol-induced apoptosis in hepatocellular carcinoma cells. Oncol Rep (2010) 23:1457-62. doi: 10.3892/ or_00000784

70. Dai W, He J, Zheng L, Bi M, Hu F, Chen M, et al. miR-148b-3p, miR-190b, and miR-429 regulate cell progression and act as potential biomarkers for breast cancer. J Breast Cancer (2019) 22:219-36. doi: 10.4048/jbc.2019.22.e19

71. Zhao Q, Liu C, Cui Q, Luan X, Wang Q, Zhou C. miR-190b promotes colorectal cancer progression through targeting forkhead box protein P2. Exp Ther Med (2019) 19:79. doi: 10.3892/etm.2019.8175

72. Pei X, Li Y, Zhu L, Zhou Z. Astrocyte-derived exosomes transfer miR-190b to inhibit oxygen and glucose deprivation-induced autophagy and neuronal apoptosis. Cell Cycle (2020) 19:906-17. doi: 10.1080/15384101.2020.1731649

Conflict of Interest: The authors declare that the research was conducted in the absence of any commercial or financial relationships that could be construed as a potential conflict of interest.

Copyright $\odot 2020$ Medina, Castillo, Liempi, Guerrero-Muñoz, Rojas-Pirela, Maya, Prieto and Kemmerling. This is an open-access article distributed under the terms of the Creative Commons Attribution License (CC BY). The use, distribution or reproduction in other forums is permitted, provided the original author(s) and the copyright owner(s) are credited and that the original publication in this journal is cited, in accordance with accepted academic practice. No use, distribution or reproduction is permitted which does not comply with these terms. 